\title{
Índice de Área Foliar de Eucalyptus Estimado por Índices de Vegetação Utilizando Imagens TM - Landsat 5
}

\author{
André Quintão de Almeida ${ }^{1}$, Aristides Ribeiro ${ }^{2}$, Rafael Coll Delgado ${ }^{3}$, \\ Yhasmin Paiva Rody ${ }^{2}$, Aline Santana de Oliveira ${ }^{2}$, Fernando Palha Leite ${ }^{4}$ \\ ${ }^{1}$ Departamento de Engenharia Agrícola - DEAGRI, Universidade Federal de Sergipe - UFS, São Cristóvão/SE, Brasil \\ ${ }^{2}$ Departamento de Engenharia Agrícola - DEA, Universidade Federal de Viçosa - UFV, Viçosa/MG, Brasil \\ ${ }^{3}$ Departamento de Ciências Ambientais - DCA, Universidade Federal Rural do Rio de Janeiro - UFRRJ, \\ Seropédica/RJ, Brasil \\ ${ }^{4}$ Celulose Nipo-Brasileira S/A - CENIBRA, Belo Oriente/MG, Brasil
}

\section{RESUMO}

Este trabalho teve como objetivo ajustar modelos de regressão entre o índice de área foliar (IAF) de plantios de Eucalyptus grandis x urophylla e índices de vegetação (IVs) de imagens TM - Landsat 5. O estudo foi realizado em plantios com diferentes idades localizados na porção mineira da bacia hidrográfica do rio Doce, entre os anos de 2008 e 2011. O IAF foi medido em campo, de forma não destrutiva, com o equipamento LAI-2000. Os IVs utilizados foram o Normalized Difference Vegetation Index (NDVI), o Soil Adjusted Vegetation Index (SAVI) e o Simple Ratio (SR). O melhor modelo foi ajustado a partir do NDVI, com coeficiente de determinação de 0,73 e erro quadrático médio da raiz de $0,37 \mathrm{~m}^{2} \mathrm{~m}^{-2}(19 \%)$. Pode-se concluir que os valores de IAF podem ser estimados pelos modelos de regressão ajustados a partir dos IVs derivados do TM - Landsat.

Palavras-chave: sensoriamento remoto orbital, análise estatística, floresta plantada.

\section{Eucalyptus Leaf Area Index Estimated by Vegetation Indices Using Landsat-5 TM Images}

\begin{abstract}
The objective of the present study was to fit regression models to the measured leaf area in eucalyptus forests and vegetation indices derived from Landsat-5 TM images. The study was carried out in commercial plantations located in the basin of the Doce River, Minas Gerais state, between 2008 and 2011. Leaf area was measured in the field, non-destructively, with the LAI-2000 device. The following indices were used: Normalized Difference Vegetation Index (NDVI), Soil Adjusted Vegetation Index (SAVI), and Simple Ratio (SR). The best model was adjusted from the NDVI, with a correlation coefficient of 0.73 and root mean square error of $0.37 \mathrm{~m}^{2} \mathrm{~m}^{-2}$ (19\%). We conclude that the leaf area index can be estimated by the regression models fit to the vegetation indices derived from the Landsat - 5 TM images.
\end{abstract}

Keywords: remote sensing, statistics, planted forest. 


\section{INTRODUÇÃO}

A área plantada de eucalipto no Brasil é de aproximadamente 4,5 milhões de ha, correspondendo a 0,5\% do território nacional. É a segunda maior nação com plantios dessa espécie. A participação do setor florestal no Produto Interno Bruto (PIB) é de 3,4\%, cerca de 44,6 bilhões de reais (SBS, 2010). Esse cenário está relacionado com a elevada produtividade $\left(>40 \mathrm{~m}^{3} \mathrm{ha}^{-1}\right.$ ano $^{-1}$ ) dos plantios brasileiros, além da crescente demanda mundial por produtos florestais advindos de reflorestamento.

O índice de área foliar (IAF) é um dos principais parâmetros fisiológicos medido em florestas de eucalipto, estando diretamente relacionado com a transpiração, a produtividade e a interceptação da precipitação pluvial. Expressa o total de área foliar por área de superfície do terreno. É também uma das principais saídas dos modelos processuais de estimativa de produtividade agrícola e florestal (Stape et al., 2004; Landsberg \& Sands, 2011). Sendo, portanto, um dos parâmetros utilizados na etapa de calibração e validação destes modelos. Em trabalho recente, Paiva (2009) utilizou o IAF na calibração e validação do modelo de produtividade 3-PG (Landsberg \& Waring, 1997) em plantios de eucalipto presentes na bacia hidrográfica do rio Doce.

O IAF pode ser obtido de forma direta, método destrutivo, ou indiretamente, método não destrutivo (Weiss et al., 2004; Bréda, 2003; Rody et al., 2014). Recentemente, métodos não destrutivos estão sendo propostos a partir de modelos empíricos de estimação, como, por exemplo, aqueles que relacionam valores de IAF com índices de vegetação (IVs) derivados de sensores remotos (Turner et al., 1999; Cohen et al., 2003; Xavier \& Vettorazzi, 2004; Monte et al., 2007; Dorigo et al., 2007; Canavesi et al., 2010). Na Argentina, Cristiano et al. (2014) analisaram a dinâmica sazonal entre os valores de IAF de plantios de Eucalyptus e do Normalized Difference Vegetation Index (NDVI) derivado do MODIS, encontrando uma boa relação entre eles. Já no Brasil, Le Maire et al. (2012) calibraram um índice específico para o monitoramento do IAF em plantios comerciais de Eucalyptus.

Os IVs realçam a componente espectral da vegetação por meio da combinação das bandas espectrais do vermelho (V) e do infravermelho próximo (IVP). Sendo mais sensíveis as variações da estrutura do dossel do que as bandas individuais. Jordan (1969) criou o índice de vegetação Simple Ratio (SR) e este tem se mostrado altamente relacionado com o IAF em florestas (Fassnacht et al., 1997; Turner et al., 1999). Representa as variações do dossel florestal a partir das respostas espectrais do mesmo nas regiões espectrais do $\mathrm{V}$ e do IVP.

O NDVI (Rouse et al., 1973) vem sendo usado para estimar o IAF em diferentes tipos de formações florestais (Fassnacht et al., 1997; Turner et al., 1999; Xavier \& Vettorazzi, 2003). Entretanto, em alguns casos, esse tem se mostrado mais sensível às variações do solo, não representando as características e as mudanças ocorridas na arquitetura do dossel vegetal. Além do problema da saturação, para elevados valores de IAF.

Na tentativa de minimizar a influência da reflectância do solo sobre o NDVI, Huete (1988) incorporou o fator L, dando origem ao índice Soil Adjusted Vegetation Index (SAVI). De acordo com as características dos solos e o tipo de cobertura, este fator pode variar entre 0 (vegetação densa) e 1 (vegetação mais rala). $O$ valor de $L$ igual a 0,5 vem sendo utilizado na maioria dos casos, independente do tipo de solo e cobertura vegetal (Washington-Allen et al., 2004).

Este trabalho teve como objetivo principal ajustar modelos de regressão entre valores medidos em campo de área foliar em florestas de E. grandis x urophylla localizado na bacia hidrográfica do rio Doce e índices de vegetação derivados de imagens TM - Landsat 5.

\section{MATERIAL E MÉTODOS}

\section{1. Área de estudo}

O trabalho foi realizado em talhões de plantios clonais de eucalipto (grandis $\mathrm{x}$ urophylla) localizados na bacia hidrográfica do rio Doce, próximos às cidades de Ipatinga e Antônio Dias. Segundo série histórica de 25 anos, de uma estação meteorológica representativa da região, o clima é caracterizado por uma precipitação média anual de $1.163 \mathrm{~mm}$, temperatura média anual do ar de $25,2{ }^{\circ} \mathrm{C}$ e umidade relativa média do ar de $65,2 \%$.

Os solos são do tipo Cambissolo Háplico Distrófico, com textura argilosa. Antes do estabelecimento do plantio manual, foi realizada subsolagem a $20 \mathrm{~cm}$ de profundidade. $\mathrm{O}$ espaçamento entre plantas foi de 3 por 3 metros e densidade de 990 árvores por hectare. 
Durante os dois primeiros anos foram realizadas limpezas (glifosato) na área para controle de ervas daninhas.

\subsection{Medidas de IAF}

O IAF foi medido em campanhas de campo, de forma não destrutiva, em seis diferentes datas, 20/8/2008, 4/11 e 16/12 de 2009, 10/5 e 16/8 de 2010 e 10/3/2011. Estudos recentes mostram uma boa concordância entre medidas de IAF realizadas pelo método destrutivo e não destrutivo (Coelho et al., 2012). Dois medidores de IAF modelo LAI-2000 (LI-COR Inc, 1992) foram utilizados simultaneamente, um abaixo dos plantios e outro em uma região aberta (branco), livre de obstáculos. O valor de IAF corresponde à média de dez leituras consecutivas abaixo do dossel florestal. As coletas foram realizadas sempre ao amanhecer e ao pôr do sol. Ao final das campanhas de campo, foram obtidos 26 valores de IAF.

\section{3. Índices de vegetação (IVs)}

Para o cálculo dos IVs foram selecionadas seis imagens (Tabela 1) do sensor Thematic Mapper (TM) a bordo do satélite Landsat 5 (ponto 73 e órbita 217) com resolução espacial de 30 metros. As datas das imagens foram 5/9/2008, 27/11 e 13/12 de 2009, $6 / 5$ e $26 / 8$ de 2010 e 25/5/2011, todas correspondentes às medidas em campo de IAF.

$\mathrm{Na}$ fase de pré-processamento, as imagens foram georreferenciadas por meio de interpolador de $1^{\circ}$ grau de alocação de vizinho mais próximo. A projeção final foi UTM/SAD69, zona 23 Sul. O erro quadrático médio encontrado para todas as imagens foi inferior a 7 metros. Para cada banda espectral do satélite Landsat 5, foi realizada a correção atmosférica, onde os níveis digitais

Tabela 1. Data de coleta do IAF e parâmetros das imagens do TM - Landsat 5 utilizadas.

Table 1. Data collection parameters of the LAI and the Landsat 5 TM images.

\begin{tabular}{|cccc|}
\hline Data IAF & $\begin{array}{c}\text { Data } \\
\text { imagem }\end{array}$ & $\begin{array}{c}\text { Elevação } \\
\left({ }^{\circ}\right)\end{array}$ & $\begin{array}{c}\text { Azimute do } \\
\text { sol }\left({ }^{\circ}\right)\end{array}$ \\
\hline $20 / 8 / 2008$ & $5 / 9 / 2008$ & 49,0 & 54,5 \\
$4 / 11 / 2009$ & $27 / 11 / 2009$ & 63,2 & 99,8 \\
\hline $16 / 12 / 2009$ & $13 / 12 / 2009$ & 61,6 & 104,1 \\
\hline $10 / 5 / 2010$ & $6 / 5 / 2010$ & 43,6 & 42,5 \\
\hline $16 / 8 / 2010$ & $26 / 8 / 2010$ & 46,8 & 49,9 \\
\hline $10 / 3 / 2011$ & $25 / 5 / 2011$ & 40,0 & 39,0 \\
\hline
\end{tabular}

foram convertidos em radiância espectral, segundo a metodologia de subtração do objeto escuro (Chavez, 1989) e posteriormente convertidos em reflectância $(\rho)$. Os valores de elevação e azimute do sol, necessários para converter a radiância em reflectância, foram obtidos diretamente das imagens (Tabela 1). Foram utilizadas duas bandas espectrais do sensor TM do satélite Landsat 5, a do vermelho $(0,63 \mu \mathrm{m}$ a $0,69 \mu \mathrm{m})$ e do infravermelho próximo $(0,76 \mu \mathrm{m}$ a $0,90 \mu \mathrm{m})$. Os índices de vegetação utilizados foram estimados a partir das duas bandas espectrais e pelas Equações 1,2 e 3 a seguir:

$$
\begin{aligned}
& N D V I=\frac{\left(\rho_{I V P}-\rho_{V}\right)}{\left(\rho_{I V P}+\rho_{V}\right)} \\
& S A V I=\frac{(1+L)\left(\rho_{I V P}-\rho_{V}\right)}{\left(L+\rho_{I V P}+\rho_{V}\right)}
\end{aligned}
$$

$S R=\frac{\rho_{I V P}}{\rho_{V}}$

em que o valor de $\mathrm{L}$ adotado foi 0,5 e $\rho$, a reflectância do pixel de cada banda, como descrito anteriormente.

Os IVs, associados pelas coordenadas geográficas (latitude e longitude) aos valores de IAF medidos em campo, correspondem à média de $3 \times 3$ pixels da imagem índice correspondente.

\subsection{Ajuste dos modelos}

Os modelos de estimativa de IAF foram ajustados a partir da análise de correlação entre as medidas do plantio em campo e os valores dos IVs. Foram utilizados modelos de regressão do tipo linear múltiplo. Depois do ajuste dos possíveis modelos, foram selecionados os com o menor Critério Bayesiano de Informação (CBI), maiores valores de $\mathrm{r}^{2}$ e menores valores de Erro Quadrado Médio da Raiz (EQMR), estimado pela Equação 4.

$E Q M R=\sqrt{\frac{\sum_{i=1}^{N}(P i-O i)^{2}}{N}}$

em que: Pi e Oi são os valores preditos e observados, respectivamente, e $\mathrm{N}$ é o número de dados avaliados.

Posteriormente, os modelos foram avaliados com uso do teste t de Student, a 5\% de probabilidade. Procedeu-se à análise dos resíduos para avaliar a existência de tendência no erro. 


\section{RESULTADOS E DISCUSSÃO}

O índice de área foliar de plantios comerciais de espécies do gênero Eucalyptus é um dos principais parâmetros fisiológicos medidos/estimados na atualidade. Além das características dendrométricas tradicionais, como Diâmetro Altura do Peito (DAP) e altura das árvores, os valores de IAF das parcelas de inventário florestal também vêm sendo medidos. Além de descrever a "qualidade" do plantio, esse índice pode estar associado à produtividade do mesmo. Modelos de estimativa da produtividade potencial da floresta, como o Physiological Principles Predicting Growth (3-PG), desenvolvido por Landsberg \& Waring (1997), utilizam o IAF como uma das suas principais entradas. Nesse estudo, buscou-se apresentar e avaliar um procedimento não destrutivo e espacial de estimativa de IAF a partir de técnicas de sensoriamento remoto e de índices de vegetação, mostrando-se como uma boa alternativa em relação aos métodos destrutivos, como pode se ver a seguir.

Os valores de IAF medidos em campo variaram entre 0,48 e $3,14 \mathrm{~m}^{2} \mathrm{~m}^{-2}$, sendo o maior valor observado na idade de 2 anos (Tabela 2). Estudos mostram que o IAF varia de forma exponencial, em função da idade do plantio, apresentando um rápido crescimento no início do ciclo de desenvolvimento. Logo após esse período verifica-se uma redução na taxa de aumento de valores, sendo essa fase coincidente com os maiores valores de área foliar. A seguir, inicia-se uma diminuição e estabilização dos valores, coincidindo com o final do ciclo comercial do plantio, aos seis e sete anos de idade (Paiva, 2009). Em plantios de eucalipto na região litorânea do Espírito Santo, Xavier et al. (2002) encontraram uma correlação positiva entre a idade do plantio e o IAF em alguns clones.

Além da idade, outro fator que interfere nos valores de área foliar são as condições climáticas locais. Estudos

Tabela 2. Valores de IAF e respectivos índices (NDVI, SAVI e SR) para os plantios de E. grandis x urophylla analisados. Table 2. LAI values and their indices (NDVI, SAVI and SR) for plantations of E. grandis $x$ urophylla.

\begin{tabular}{|c|c|c|c|c|}
\hline Idade plantio (anos) & $\operatorname{IAF}\left(m^{2} m^{-2}\right)$ & NDVI & SAVI & SR \\
\hline 0,5 & 0,48 & 0,33 & 0,20 & 2,00 \\
\hline 1 & 2,17 & 0,78 & 0,50 & 7,74 \\
\hline 1,3 & 2,43 & 0,66 & 0,41 & 4,80 \\
\hline 1,4 & 2,51 & 0,76 & 0,66 & 7,71 \\
\hline 2 & 3,14 & 0,79 & 0,51 & 8,20 \\
\hline 4 & 2,09 & 0,71 & 0,42 & 5,82 \\
\hline 4 & 1,99 & 0,75 & 0,51 & 7,11 \\
\hline 4 & 2,35 & 0,75 & 0,50 & 7,09 \\
\hline 4 & 2,63 & 0,73 & 0,50 & 6,45 \\
\hline 4 & 2,38 & 0,76 & 0,50 & 7,56 \\
\hline 4 & 2,45 & 0,74 & 0,47 & 6,95 \\
\hline 4 & 2,25 & 0,70 & 0,46 & 5,61 \\
\hline 4 & 2,02 & 0,76 & 0,51 & 7,32 \\
\hline 4 & 2,14 & 0,76 & 0,50 & 7,22 \\
\hline 5 & 1,83 & 0,72 & 0,42 & 6,29 \\
\hline 5 & 2,37 & 0,71 & 0,41 & 5,97 \\
\hline 5 & 1,76 & 0,58 & 0,32 & 3,85 \\
\hline 5 & 1,44 & 0,72 & 0,42 & 6,26 \\
\hline 5 & 2,33 & 0,66 & 0,36 & 4,86 \\
\hline 6 & 2,34 & 0,72 & 0,47 & 6,13 \\
\hline 6 & 2,90 & 0,75 & 0,46 & 6,85 \\
\hline 7 & 2,85 & 0,74 & 0,46 & 6,70 \\
\hline 7 & 2,52 & 0,75 & 0,46 & 7,10 \\
\hline 7 & 1,61 & 0,72 & 0,32 & 6,05 \\
\hline 7 & 1,06 & 0,53 & 0,29 & 3,22 \\
\hline 7 & 1,88 & 0,71 & 0,36 & 5,95 \\
\hline
\end{tabular}


mostram (Souza et al., 2006) que a produtividade em plantios de eucalipto em regiões com menor déficit de pressão de vapor d'água é maior, podendo essa estar representada por elevados índices de área foliar.

Os valores dos IVs variaram em função dos valores de IAF, quanto maior a área foliar, maior os valores dos índices. Isso expressa a relação sempre positiva entre esse parâmetro biofísico da espécie e os índices utilizados, comprovada pelos valores de coeficiente de correlação apresentados na primeira linha da Figura 1 e pelos histogramas de classes de valores da diagonal principal da mesma figura. Os valores de NDVI, SAVI e SR variaram entre 0,33 e 0,79, 0,20 e 0,66 e 2,00 e 8,20, respectivamente. O índice Simple Ratio (SR) apresentou o maior valor $(0,81)$ de coeficiente de correlação. Assim como o NDVI, este índice é estimado de forma bem simples, envolvendo duas bandas do espectro eletromagnético (vermelho e do infravermelho próximo) altamente correlacionadas com a vegetação (Turner et al., 1999). Todas as correlações foram positivas, evidenciando que o aumento dos valores de IAF foi acompanhado por um aumento dos valores do IVs.

Ao se analisar o diagrama de dispersão (primeira coluna da Figura 1) entre os valores de IAF e os índices analisados, nota-se uma clara saturação dos dados orbitais, principalmente para o NDVI e o SAVI. Tal comportamento ocorre em decorrência da completa absorção da radiação na região do vermelho por parte da clorofila e carotenos presentes nas folhas,

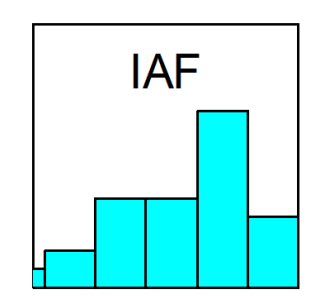

$\begin{array}{llll}0,4 & 0,5 & 0,6 & 0,7\end{array}$
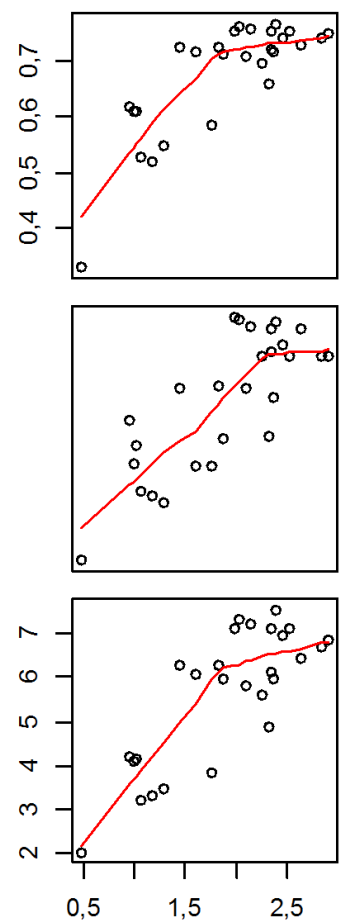
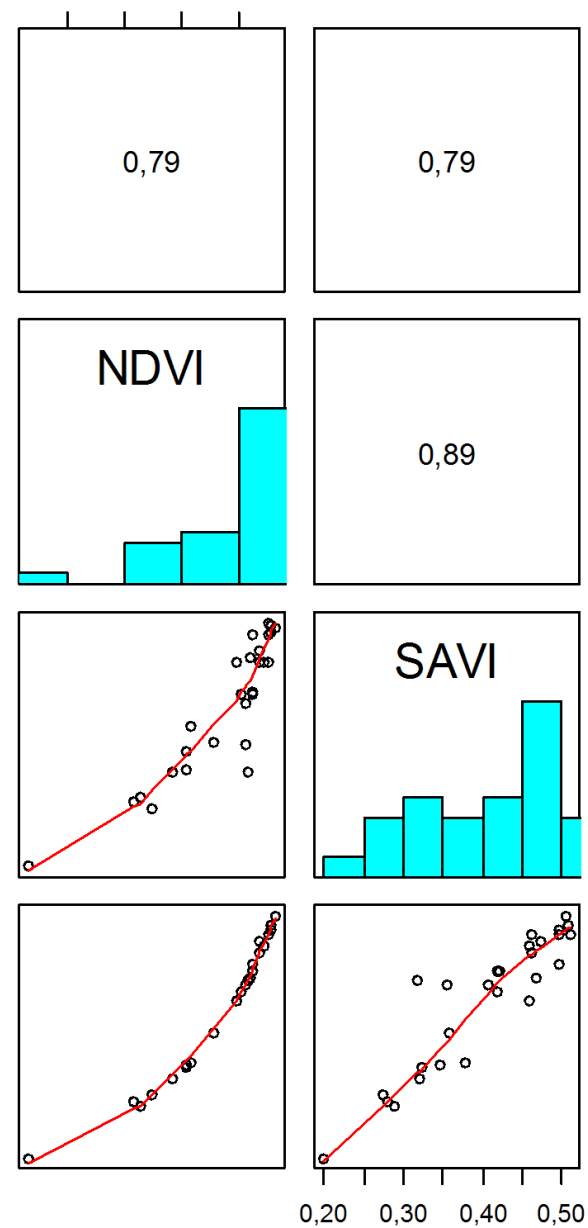
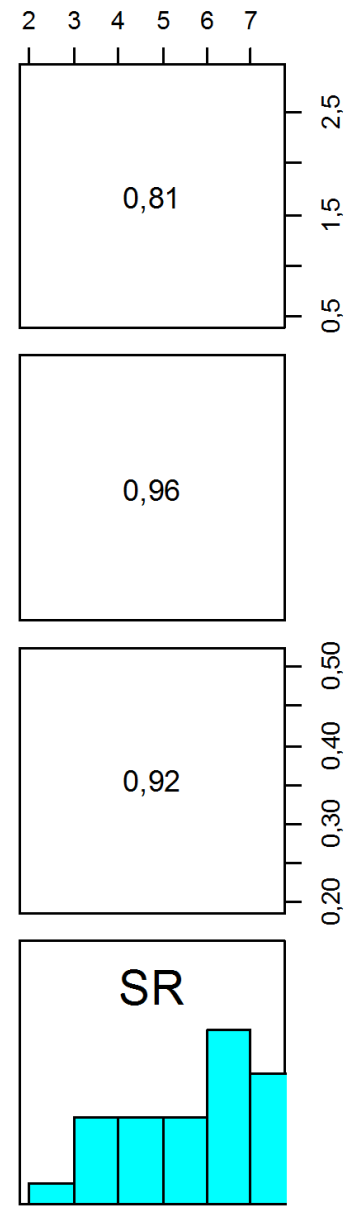

Figura 1. Diagrama de dispersão e coeficientes de correlação entre os valores de IAF e os IVs extraídos do sensor TM.

Figure 1. Scatter plot and correlation coefficients between LAI and IV's extracted from the TM sensor. 
a partir de um determinado valor de IAF (Liang, 2004). Comportamento semelhante foi encontrado em outros trabalhos com a mesma espécie florestal (Xavier \& Vettorazzi, 2003, 2004). A saturação dos dados TM em estudos que buscam extrair características da vegetação (i.e., IAF, diâmetro, altura, biomassa etc.) é uma das limitações deste produto. Estudos recentes demonstram um grande potencial de outras técnicas na estimativa de parâmetros dendrométricos da vegetação, como Light Detection And Ranging (LIDAR). Não ocorrendo, por exemplo, a saturação desses dados em vegetações mais densas.

O mesmo comportamento foi encontrado por Turner et al. (1999), que relacionaram índices de vegetação derivados de imagens TM - Landsat com valores de área foliar em espécies florestais de clima temperado. Isso expressa a relação sempre positiva entre o IAF e os índices utilizados, calculados a partir das bandas do vermelho e do infravermelho próximo. Regiões do espectro com menores e maiores valores de reflectância em áreas cobertas por florestas, resultado da interação da radiação eletromagnética com os pigmentos fotossintéticos presentes nas folhas.

Os melhores modelos (Tabela 3 ) foram ajustados pela relação linear entre o $\log$ do IAF e os índices de vegetação. Os modelos ajustados apresentaram bom desempenho, com elevados valores de coeficiente de determinação $\left(\mathrm{r}^{2}>0,6\right)$ e baixos valores de EQMR $(<22 \%)$ e do CBI (Tabela 3). A análise dos resíduos confirmou a inexistência de tendência na estimativa dos erros. Os melhores modelos foram ajustados considerando a relação linear simples com NDVI e a relação linear múltipla com o NDVI e o SAVI (Tabela 3). Na relação linear dos índices com o IAF os valores do $r^{2}(0,6-0,75)$ são semelhantes aos encontrados em outros trabalhos, como o realizado por Fassnacht et al. (1997), em plantios de coníferas no norte de Wisconsin. De maneira geral, o NDVI é mais sensível à presença de clorofila e outros pigmentos responsáveis pela absorção da radiação solar, na banda do vermelho (Gao et al., 2000; Huete et al., 1997).

Apesar do elevado valor de $r^{2}(0,68)$, o modelo ajustado a partir dos valores de SAVI apresentou o maior EQMR dentre os IVs analisados. O mesmo também foi observado por Xavier \& Vettorazzi (2004). Esse resultado pode estar associado ao valor do fator L utilizado, igual a 0,5 . Sugere-se, que em trabalhos futuros, seja ajustado o valor desse fator para a região de estudo, conseguindo dessa forma introduzir a influência do substrato e, consequentemente, encontrar uma maior relação desse índice com os valores de IAF.

A relação linear do IAF com o SR foi a que apresentou a maior correlação $(0,81)$, indicando que, mesmo nos plantios com valores elevados de $\operatorname{IAF}(>2,5)$, os valores de SR não saturaram muito (Figura 1). Diferente do que foi observado na relação com o NDVI e o SAVI. O modelo linear múltiplo, considerando como variáveis explicativas o NDVI e o SAVI, conseguiu explicar cerca de $73 \%$ das variações dos valores de IAF. Assim como o modelo linear simples em função do NDVI, os valores de EQMR também foram baixos, $0,37 \mathrm{~m}^{2} \mathrm{~m}^{-2}$ (19\%).

A Figura 2 apresenta os diagramas de dispersão entre os valores medidos e estimados de IAF. Apesar da saturação dos dados orbitais, os diagramas de dispersão mostram que os modelos ajustados conseguiram estimar os valores de IAF para os plantios analisados.

Os resultados encontrados neste estudo confirmam os apresentados em outros trabalhos. Mostrando que as técnicas de sensoriamento remoto e os índices de vegetação derivados do sensor TM podem ser utilizados para a estimativa do IAF de florestas comerciais do gênero Eucalyptus na área de estudo. Apresentando como vantagens, em relação aos métodos tradicionais (destrutivos), uma estimativa rápida, confiável e espacial desse parâmetro tão importante no monitoramento da vegetação. Nos próximos estudos, sugere-se que novas técnicas sejam avaliadas, além de imagens de satélite com uma maior resolução espacial.

Tabela 3. Resumo da análise de regressão da relação entre o IAF e os índices NDVI, SAVI e SR.

Table 3. Summary of regression analysis of the relationship between LAI and NDVI, SAVI and SR indices.

\begin{tabular}{ccccc}
\hline Modelo (Log IAF) & $\mathbf{r}^{\mathbf{2}}$ & EQMR $\left(\mathbf{m}^{\mathbf{2}} \mathbf{~ m}^{-\mathbf{2}}\right)$ & $\mathbf{C B I}$ & Valor $\mathbf{p}$ \\
\hline 3,6 (NDVI) - 1,9 & 0,73 & $0,37(19 \%)$ & -30 & $<0,01$ \\
3,93 (SAVI) - 1,02 & 0,68 & $0,42(22 \%)$ & -25 & $<0,01$ \\
\hline 0,23 (SR) - 0,73 & 0,62 & $0,40(21 \%)$ & -20 & $<0,01$ \\
3,06 (NDVI) + 0,72 (SAVI) -1,8 & 0,73 & $0,37(19 \%)$ & -27 & $<0,01$ \\
\hline
\end{tabular}



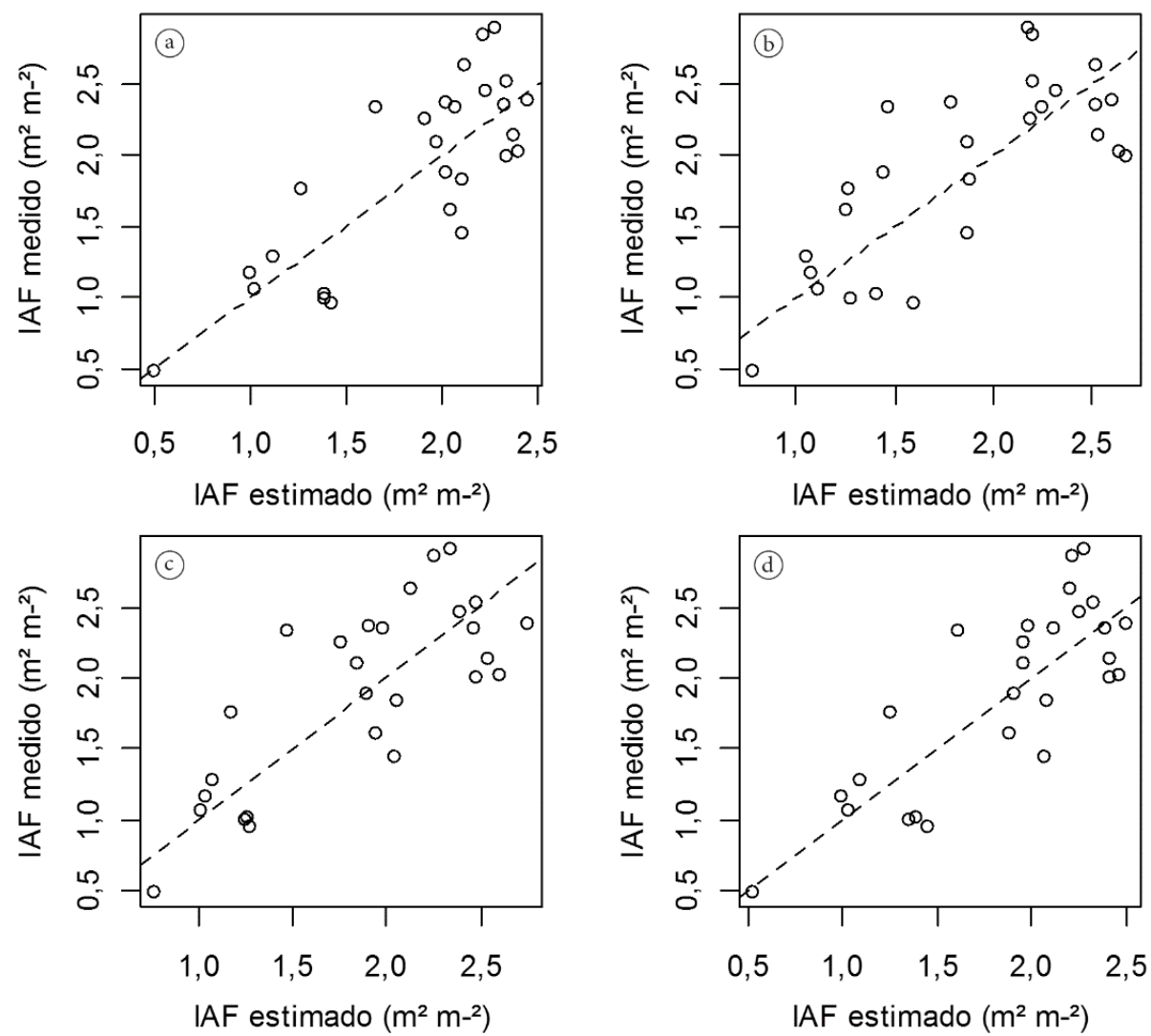

Figura 2. Diagrama de dispersão entre os valores de IAF $\left(\mathrm{m}^{2} \mathrm{~m}^{-2}\right)$ medidos e estimados pelos modelos de regressão ajustados para plantios de Eucalyptus; A, NDVI; B, SAVI; C, SR; e D, NDVI + SAVI.

Figure 2. Scatter plot between LAI $\left(\mathrm{m}^{2} \mathrm{~m}^{-2}\right)$ measured and estimated by regression models adjusted for Eucalyptus. A, NDVI; B, SAVI; C, SR; and D, NDVI + SAVI.

\section{CONCLUSÕES}

Os modelos de regressão ajustados indicaram significativa relação entre os valores de IAF em plantios de E. grandis x urophylla e os índices de vegetação calculados a partir das imagens TM - Landsat 5.

Apesar da saturação dos valores de NDVI, os modelos ajustados a partir desse índice apresentaram os melhores resultados, podendo ser utilizados para estimar os valores de IAF dos plantios analisados.

O SAVI teve um alto valor do coeficiente de determinação, mas será necessário em trabalhos futuros corrigir a constante de efeitos do solo para esta área de estudo, devido aos maiores valores de erro quadrático médio encontrado.
Fazem-se necessários estudos futuros com outras plataformas, sensores orbitais, outros índices de vegetação, além de uma análise sazonal, para que se possa inferir sobre a precisão e correlação dos dados.

\section{STATUS DA SUBMISSÃO}

Recebido: 29 jun., 2014

Aceito: 21 mar., 2015

\section{AUTOR(ES) PARA CORRESPONDÊNCIA}

\section{André Quintão de Almeida}

Departamento de Engenharia Agrícola -

DEAGRI, Universidade Federal de Sergipe - UFS, São Cristóvão, SE, Brasil

e-mail: andreqa@gmail.com 


\section{REFERENNCIAS}

Bréda NJJ. Ground-based measurements of leaf area index: a review of methods, instruments and current controversies. Journal of Experimental Botany 2003; 54(392): 2403-2417. http://dx.doi.org/10.1093/jxb/erg263. PMid:14565947.

Canavesi V, Ponzoni FJ, Valeriano MM. Estimativa de volume de madeira em plantios de eucalyptus spp. utilizando dados hiperespectrais e dados topográficos. Revista Árvore 2010; 34(3): 539-549. http://dx.doi.org/10.1590/ S0100-67622010000300018.

Chavez PS Jr. Radiometric calibration of Landsat thematic mapper multispectral images. Photogrammetric Engineering and Remote Sensing 1989; 55: 1285-1294.

Coelho MA Fo, Villa-Nova NA, Angelocci LR, Marin FR, Righi CA. Método para estimativa do IAF de árvores isoladas ou de plantações com dossel fechado. Revista Brasileira de Engenharia Agrícola e Ambiental 2012; 16(5): 529-538. http://dx.doi.org/10.1590/S1415-43662012000500009.

Cohen WB, Maiersperger TK, Gower ST, Turner DP. An improved strategy for regression of biophysical variables and Landsat ETM+ data. Remote Sensing of Environment 2003; 84(4): 561-571. http://dx.doi.org/10.1016/S00344257(02)00173-6.

Cristiano PM, Madanes N, Campanello PI, di Francescantonio D, Rodríguez SA, Zhang YJ et al. High NDVI and potential canopy photosynthesis of south american subtropical forests despite seasonal changes in leaf area index and air temperature. Forests 2014; 5(2): 287-308. http://dx.doi. org/10.3390/f5020287.

Dorigo WA, Zurita-Milla R, de Wit AJW, Brazile J, Singh R, Schaepman ME. A review on reflective remote sensing and data assimilation techniques for enhanced agroecosystem modeling. International Journal of Applied Earth Observation and Geoinformation 2007; 9(2): 165193. http://dx.doi.org/10.1016/j.jag.2006.05.003.

Fassnacht KS, Gower ST, Mackenzie MD, Nordheim EV, Lillesand TM. Estimating the leaf area index of north central Wisconsin forests using the Landsat thematic mapper. Remote Sensing of Environment 1997; 61(2): 229245. http://dx.doi.org/10.1016/S0034-4257(97)00005-9.

Gao X, Huete AR, Ni WG. MIura T. Optical-biophysical relationships of vegetation spectra without background contamination. Remote Sensing of Environment 2000; 74(3): 609-620. http://dx.doi.org/10.1016/S0034-4257(00)00150-4.

Huete AR, Liu HQ, Batchily K, Leeuwen WV. A Comparison of vegetation indices over a global set of TM images for EOS-MODIS. Remote Sensing of Environment 1997; 59(3): 440-451. http://dx.doi.org/10.1016/S0034-4257(96)00112-5.

Huete AR. A soil-adjusted vegetation index (SAVI). Remote Sensing of Environment 1988; 25(3): 295-309. http://dx.doi. org/10.1016/0034-4257(88)90106-X.
Jordan CF. Derivation of leaf-area index from quality of light on the forest floor. Ecology 1969; 50(4): 663-666. http://dx.doi.org/10.2307/1936256.

Landsberg JJ, Sands P. Physiological ecology of forest production: principles, processes and models. New York: Academic Press; 2011. 352 p. Terrestrial Ecology Series n. 4.

Landsberg JJ, Waring RH. A generalised model of forest productivity using simplified concepts of radiation-use efficiency, carbon balance and partitioning. Forest Ecology and Management 1997; 95(3): 209-228. http://dx.doi. org/10.1016/S0378-1127(97)00026-1.

Le Maire G, Marsden C, Nouvellon Y, Stape JL, Ponzoni FJ. Calibration of a species-specific spectral vegetation index for leaf area index (LAI) monitoring: example with MODIS reflectance time-series on Eucalyptus plantations. Remote Sensing 2012; 4(12): 3766-3780. http://dx.doi. org/10.3390/rs4123766.

Liang S. Quantitative remote sensing of land surfaces. New Jersey, USA: Wiley Interscience; 2004. 534 p.

LI-COR Inc. LAI-2000 plant canopy analyzer: instruction manual. Nebraska: LI-COR, Inc.; 1992. 166 p.

Monte MA, Reis MGF, Reis GG, Leite HG, Stocks JJ. Métodos indiretos de estimação da cobertura de dossel em povoamentos de clone de eucalipto. Pesquisa Agropecuaria Brasileira 2007; 42(6): 769-775. http://dx.doi.org/10.1590/ S0100-204X2007000600002.

Paiva YG. Estimativa do índice de área foliar por métodos óticos e sensoriamento remoto para calibrar modelo ecofisiológico em plantios de eucalipto em áreas de relevo ondulado [dissertação]. Viçosa: Universidade Federal de Viçosa; 2009. 62 p.

Rody HP, Ribeiro A, Pezzopane JEM, Gleriani JM, Almeida $A Q$, Leite FP. Estimates of the leaf area index (LAI) using LAI-2000 and hemispherical photos in Eucalyptus plantations. Ciência Florestal 2014; 4: 923-932.

Rouse JW, Haas RH, Schell JA, Deering DW. Monitoring vegetation systems in the great plains with ERTS. In: $3 r d$ ERTS Symposium, NASA SP-351 I. Washington, D.C.: NASA; 1973. 309-317.

Sociedade Brasileira de Silvicultura - SBS. Fatos e números do Brasil florestal [online]. [acesso em 2010 fev. 10]. Disponível em: http://www.sbs.org.br/ FatoseNumerosdoBrasilFlorestal.pdf

Souza MJH, Ribeiro A, Leite HG, Leite FP, Minuzzi RB. Disponibilidade hídrica do solo e produtividade do eucalipto em três regiões da Bacia do Rio Doce. Revista Árvore 2006; 30(3): 399-410. http://dx.doi.org/10.1590/ S0100-67622006000300010.

Stape JL, Ryan MG, Binkley D. Testing the utility of the 3-PG model for growth of Eucalyptus grandis-urophylla with natural and manipulated supplies of water and nutrients. Forest Ecology and Management 2004; 193: 219-234. http://dx.doi.org/10.1016/j.foreco.2004.01.031. 
Turner DP, Cohen WB, Kennedy RE, Fassnacht KS, Briggs JM. Relationships between leaf area index and Landsat TM Spectral Vegetation Indices across three temperate zone sites. Remote Sensing of Environment 1999; 70(1): 52-68. http://dx.doi.org/10.1016/S0034-4257(99)00057-7.

Washington-Allen RAW, Ramsey RD, West NE. Spatiotemporal mapping of the dry season vegetationresponse of sagebrush steppe. Community Ecology 2004; 5(1): 69-79. http:// dx.doi.org/10.1556/ComEc.5.2004.1.7.

Weiss M, Baret F, Smith GJ, Jonckheere I, Coppin P. Review of methods for in situ leaf area index (LAI) determination. Agricultural and Forest Meteorology 2004; 121(1-2): 3753. http://dx.doi.org/10.1016/j.agrformet.2003.08.001.
Xavier AC, Soares JV, Almeida AC. Variação do índice de área foliar em clones de eucalipto ao longo de seu ciclo de crescimento. Revista Árvore 2002; 26(4): 421-427. http:// dx.doi.org/10.1590/S0100-67622002000400004.

Xavier AC, Vettorazzi CA. Leaf area index of ground covers in a subtropical watershed. Scientia Agricola 2003; 60(3): 425-431. http://dx.doi.org/10.1590/S010390162003000300002 .

Xavier AC, Vettorazzi CA. Mapping leaf area index through spectral vegetation indices in a subtropical watershed. International Journal of Remote Sensing 2004; 25(9): 16611672. http://dx.doi.org/10.1080/01431160310001620803. 